\title{
Study on the color difference of Pomelo cv. Huangjinmiyou in different planting areas
}

\author{
Yuan Yao ${ }^{1}$, Wenxin Xu${ }^{1}$, Jiaqi Wang ${ }^{1}$, Bozhi Wang ${ }^{1}$, Xiaoyu Tang ${ }^{1}$, Tiantian Dong ${ }^{1}$, Bo Xiong ${ }^{1}$, Guochao Sun ${ }^{1}$, Ling Liao ${ }^{1}$, \\ Siya $\mathrm{He}^{1}$, and Zhihui Wang ${ }^{2 *}$ \\ ${ }^{1}$ College of Horticulture, Sichuan Agricultural University, Chengdu, Sichuan, 611130, China \\ ${ }^{2}$ Institute of Pomology \& Olericulture, Sichuan Agricultural University, Chengdu, Sichuan, 611130, China
}

\begin{abstract}
In order to study the effect of different planting areas on the color of Pomelo cv. Huangjinmiyou fruit, Pujiang and Guang'an Pomelo cv. Huangjinmiyou were used in this experiment. Brightness, red and green deviation and blue and yellow deviation of exocarp, mesocarp and pulp parts in the test samples were measured, and the composite index of color difference was calculated. The results showed that the comprehensive index of color difference in the exocarp and pulp of Pomelo cv. Huangjinmiyou from the two planting areas changed almost synchronously. But it was negative at 90 days after anthesis and positive at 120 days after anthesis in the mesocarp of Pujiang pomelo. It was still negative at 150 days after anthesis in the mesocarp of Guang'an pomelo until 180 days after anthesis. It reflects the comprehensive index of fruit color displayed by the accumulation of carotenoids. The external environment stimulates the accumulation of carotenoids. Therefore, color variation difference may be caused by different environmental factors in the two regions.
\end{abstract}

\section{Introduction}

Pomelo cv. Huangjinmiyou is a new bud variety of guanxi pomelo[1]. And it is selected by the Fruit Research Institute of Fujian Academy of Agricultural Sciences and Pinghe economic work station [2]. Fruit color is an important index to evaluate the quality of fruit. Studying on the color of Pomelo cv. Huangjinmiyou is beneficial to improve the color of fruit, the appearance quality of fruit and obtain greater economic benefits. The changes of main pigment substances during fruit ripening include the synthesis of carotenoids, chlorophyll degradation, etc[3]. Its components and contents determine the color and commercial quality of fruit[4-6]. Therefore, even if the same variety is planted in different areas, apart from the production technology and other factors, there will be differences in the color of Pomelo cv. Huangjinmiyou fruit due to environmental factors. The external environment will stimulate the accumulation of carotenoids[7-8]. The comprehensive index of color difference reflects the comprehensive index of fruit color displayed by the accumulation of carotenoids[9]. Therefore, this experiment will study the color difference of Pomelo cv. Huangjinmiyou in different places through the influence of environmental factors on the brightness, blue and yellow deviation and red and green deviation that made up the comprehensive index of color difference.

\section{Materials and methods}

\subsection{Materials}

There are two origins of Pomelo cv. Huangjinmiyou: Shouan in Pujiang(PP) and Qianfeng in Guang'an(GP). Four sampling stages were set up: 90 days after anthesis, 120 days after anthesis, 150 days after anthesis and 180 days after anthesis. After harvest, they were transported to the laboratory, and the fruits with large uniformity and uniform maturity were selected as the samples.

\subsection{Measurement indicators and methods}

The color of fruit was determined by the color difference instrument (Hunter associates laboratory, Inc. , USA), and the CIE (International Commission on illumination) $1976 \mathrm{~L} * \mathrm{a} * \mathrm{~b} *$ color difference system was used as the standard. L* (brightness), a * (red and green deviation), $\mathrm{b} *$ (blue and yellow deviation), calculation formula: color difference comprehensive index CCI (CCI $=1000$ $\times \mathrm{a} * /(\mathrm{L} * \times \mathrm{b} *)$. The positive value of $\mathrm{CCI}$ indicates the degree of red and yellow, and the negative value indicates the degree of blue and green (Zhou et al. , 2010)[12]. In four periods, six grapefruits were measured each time, three points were selected for each grapefruit, and the average value of six data was one repetition, totally three repeats.

\footnotetext{
*Corresponding author's e-mail: wangzhihui318@126.com
} 


\section{Results and analysis}

\subsection{Changes of CCI in the exocarp}

It can be seen from Fig 1 that the CCI values of exocarp of PP and GP were almost synchronous in different periods after anthesis, and they were all negative. Both of them showed a trend of increasing CCI value with the post anthesis period. The results showed that the blue-green degree of exocarp decreased gradually after anthesis. The blue-green degree of PP exocarp was slightly higher than that of GP within 150 DAA and a little lower than 180 DAA.

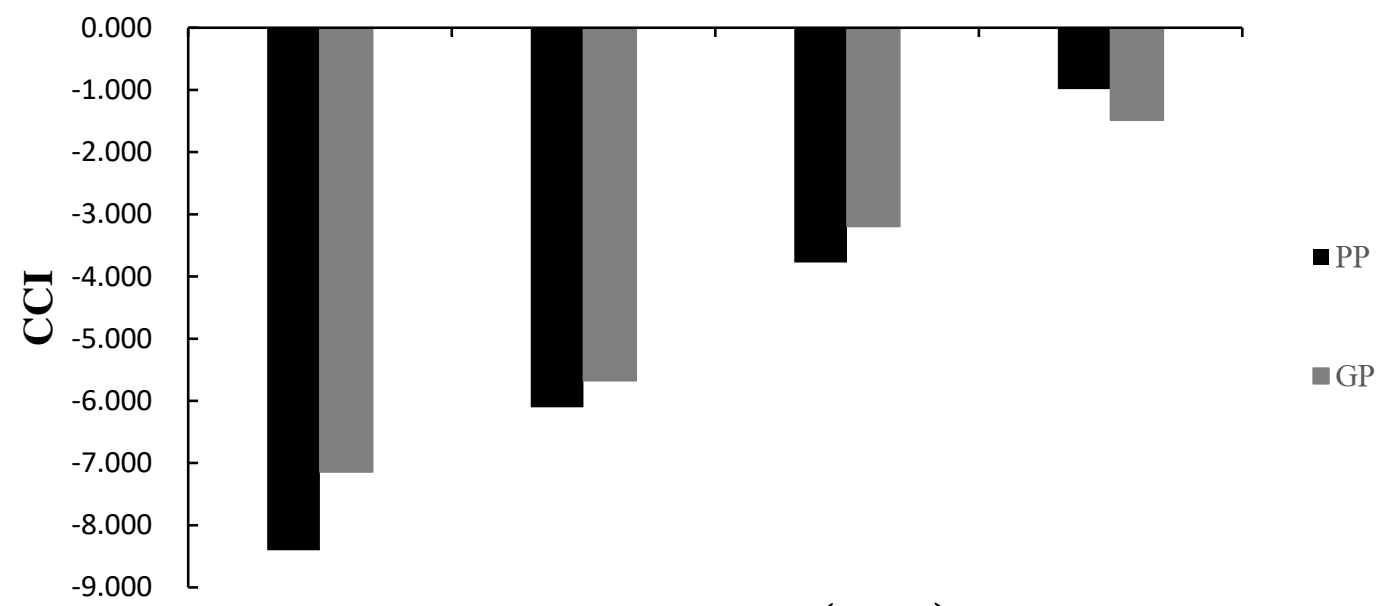

\section{Days after anthesis（DAA）}

Fig1. Changes of CCI value in exocarp of PP and GP pomelo in different DAA mesocarp of GP was still negative 150 days after anthesis, and turned positive 180 days after anthesis, indicating that its color was in red and yellow stage. The CCI value

\subsection{Changes of $\mathrm{CCl}$ in the mesocarp}

It can be seen from Fig 2 that the CCI value of Pujiang Shouan pomelo was negative at $90 \mathrm{DAA}$, and positive at 120 DAA with keeping growing. The CCI value of

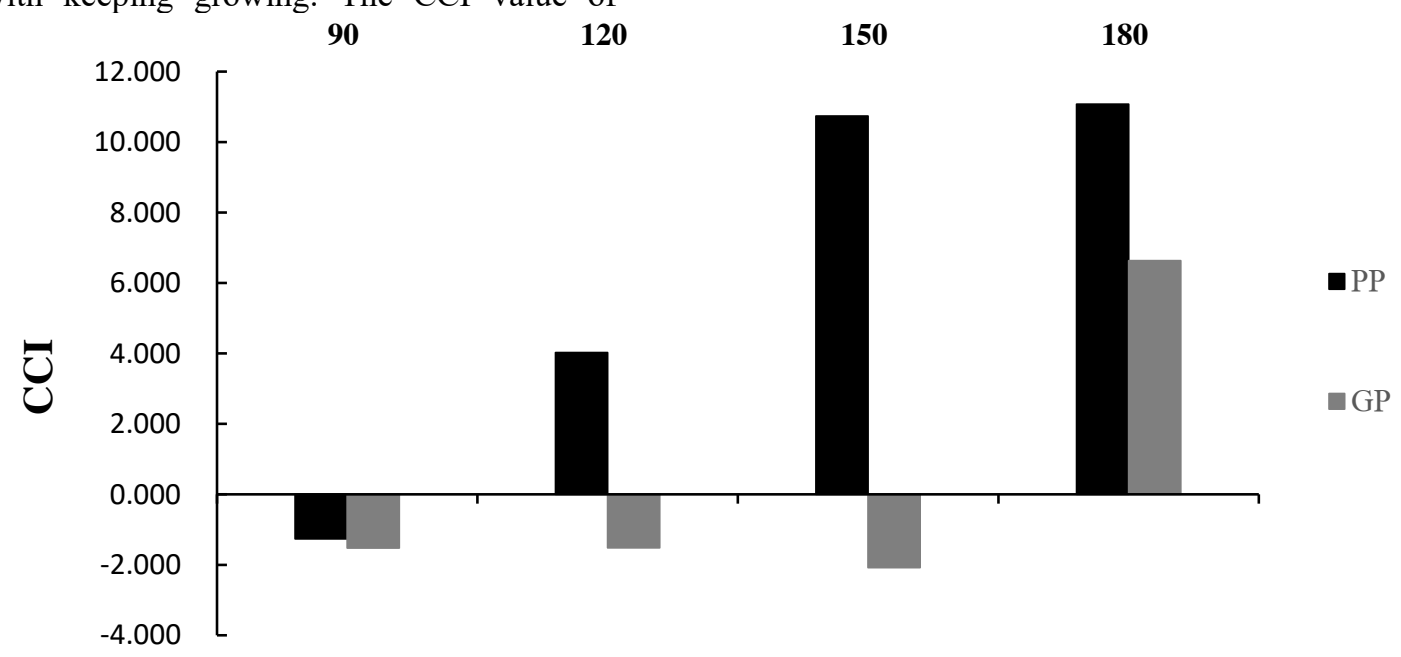

\section{Days after anthesis (DAA)}

Fig2. Changes of CCI value in mesocarp of PP and GP pomelo in different DAA low level, and 12. 447 at 120 DAA. After that, the degree of red and yellow decreased, but all of them were at a

\subsection{Changes of $\mathrm{CCl}$ in the pulp}

It can be seen from Figure 3 that the CCI values of PP and GP pulp were both positive values 90 DAA. The CCI value of PP was 1.255 at 90 DAA which was at a high level. The CCI value of GP reached 9.477 at 90 DAA, and increased slightly with the increase of anthesis period, reaching 12.873 at 180 DAA. 


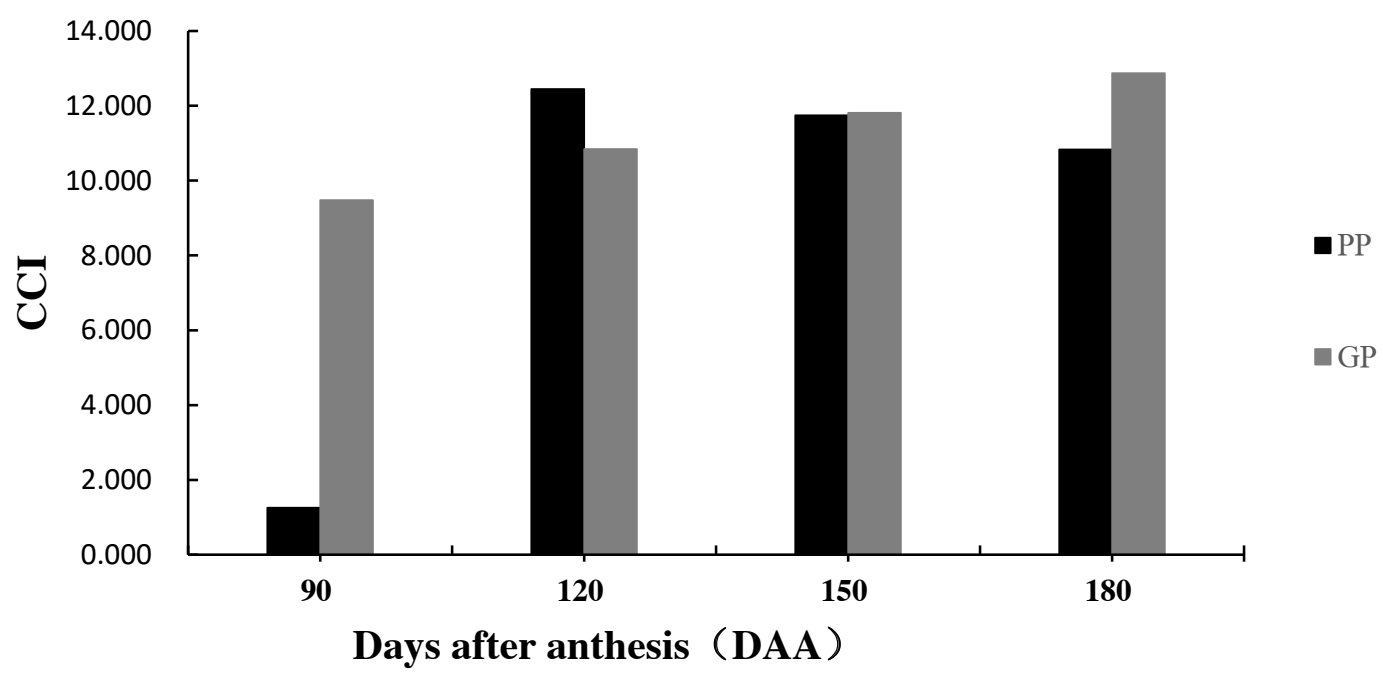

Fig3. Changes of CCI value in pulp of PP and GP in different DAA

\subsection{Changes of $L$ * in the exocarp, mesocarp and pulp}

It can be seen from Fig. 4 that the $\mathrm{L}^{*}$ values of exocarp, mesocarp and pulp of the two varieties change almost synchronously in different DAA. The $L^{*}$ value of exocarp increased with the DAA, and the $\mathrm{L}^{*}$ value of GP was slightly higher than that of PP in each period. In addition, the $\mathrm{L}^{*}$ value of mesocarp and pulp decreased with the increase of post anthesis period, and the value of mesocarp and pulp of PP was slightly higher than that of GP.

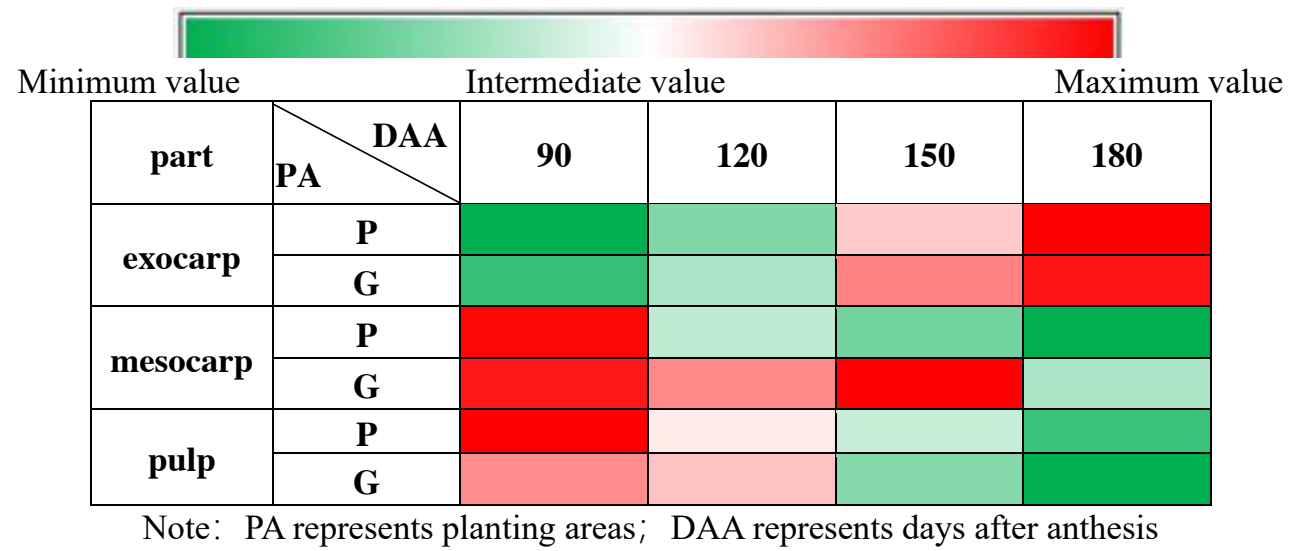

Fig4. The variation of $\mathrm{L}^{*}$ values of exocarp, mesocarp and pulp between PP and GP

\subsection{Changes of a * in the exocarp, mesocarp and pulp}

$\mathrm{a}^{*}$ positive value represents red, negative value represents green. The a* values of exocarp were all negative. The absolute value of a* decreased with the increase of post anthesis period, indicating that the green degree of exocarp decreased gradually after anthesis. The absolute value of $\mathrm{a}^{*}$ of GP was slightly higher than that of PP. The a * values of the mesocarp layer of PP were negative at 90 days after flowering, positive at 120 days after flowering, and gradually increased. The red green deviation of GP was still negative 150 days after flowering. a* values of both pulp parts were positive, the values of PP tended to be stable at 120 DAA, while the values of GP gradually decreased with the increase of time after flowering, and the red degree gradually weakened.

\begin{tabular}{|c|c|c|c|c|c|}
\hline \multirow{2}{*}{ part } & PA & 90 & 120 & 150 & 180 \\
\hline \multirow{2}{*}{ exocarp } & P & & & & \\
\hline \multirow{2}{*}{ mesocarp } & G & & & & \\
\hline & P & & & & \\
\hline \multirow{2}{*}{ pulp } & P & & & & \\
\hline & G & & & & \\
\hline
\end{tabular}

Fig5. The variation of a * values of exocarp, mesocarp and pulp between PP and GP 


\subsection{Changes of $b$ * in the exocarp, mesocarp and pulp}

$b^{*}$ positive value represents yellow, negative value represents blue, the following values are all positive. It can be seen from Fig. 6 that the $b *$ values of the exocarp parts of Pomelo cv. Huangjinmiyou from the two planting areas gradually increased with the increase of time after flowering, and the difference between the two values was not significant. The $\mathrm{b} *$ of PP white skin changed little in different periods, the minimum value was 7. 943, the maximum value was 9. 143, the minimum value of the $\mathrm{b} *$ of GP was 8. 057, the maximum value was 10.917 . From 90 to 150 DAA, the $\mathrm{b}^{*}$ of the pulp of PP gradually decreased, and increased at $180 \mathrm{DAA}$. From 90 to $180 \mathrm{DAA}$, the $\mathrm{b}^{*}$ values in the pulp of GP decreased.

\begin{tabular}{|c|c|c|c|c|c|}
\hline part & PA DAA & 90 & 120 & 150 & 180 \\
\hline \multirow{2}{*}{ exocarp } & $\mathbf{P}$ & & & & \\
\hline & G & & & & \\
\hline \multirow{2}{*}{ mesocarp } & $\mathbf{P}$ & & & & \\
\hline & G & & & & \\
\hline \multirow{2}{*}{ pulp } & $\mathbf{P}$ & & & & \\
\hline & G & & & & \\
\hline
\end{tabular}

Fig6. The variation of $\mathrm{b}$ * values of exocarp, mesocarp and pulp between PP and GP

\section{Conclusion}

In this experiment, through the study on the changes of color index in pomelo fruit after anthesis, the following conclusions are drawn. It can be seen from the changes of color indexes of PP and GP exocarp that the environment mainly affects their $\mathrm{L} *$ and $\mathrm{a} *$ while the difference of $\mathrm{b} *$ between them is not obvious. The $\mathrm{L} *$ and $\mathrm{a} *$ values of PP and GP were different from anthesis. Within 180 DAA, the $\mathrm{L} *$ value of GP was about 1.81-4.76 higher than that of PP, and the a * value was about $0.07-1.55$ lower than that of PP. This result is consistent with the fact that the color of GP exocarp is more orange than PP exocarp. It can be seen from the changes of color indexes of PP and GP pulp that environment has a great influence on $\mathrm{L} *, \mathrm{a} *, \mathrm{~b} *$. The $\mathrm{L}$ $*, a *, b *$ values of GP began to decrease after anthesis, but the CCI values showed a trend of increasing. The $\mathrm{L}^{*}$ value of PP decreased gradually after anthesis, but its a * value was significantly different from GP at 90 days after anthesis, and showed a trend of first increasing, then decreasing and then increasing after anthesis. And $b *$ value first decreased and then increased 150 days after anthesis. There was no significant difference inmesocarp color between GP and PP. Temperature, light and other factors can affect the color of fruit[10]. In addition to environmental factors, the study showed that bagging and bagging time also had an effect on the appearance and physical and chemical properties of Pomelo cv. Huangjinmiyou.

\section{References:}

1. Lin Yanjin et al. The effect of bagging period on the appearance and endoplasm of golden pomelo fruit. Fruit trees of southern China, 2016. 45 (06): 47-48.

2. Lu Xinkun, et al. High quality and high efficiency cultivation technology of golden pomelo.
3. Tang Hong et al. Research progress on molecular mechanism of plant hormone regulating fruit color formation. Molecular plant breeding, 2019. 17 (08): 2705-2711

4. Yang Xiangyan, Cai Yuanbao, sun Guangming. The relationship between the formation of pineapple flesh color and the change of carotenoid components [J]. Journal of fruit trees, 2010, 27 (1): 135-139.

5. Huang Bincheng, Ji Jing, Wang Gang, et al. Research progress of plant carotenoids [J]. Tianjin Agricultural Science, 2006, 12 (2): 13-17.

6. Fraser P D, Bramley P M.The biosynthesis and nutritional uses of carotenoids[J]. Progress in lipid research,2004,43(3):228-265.

7. Zhang L, Ma G, Yamawaki K, et al. Effect of blue LED light intensity on carotenoid accumulation in citrus juice sacs[J]. Journal of Plant Physiology, 2015, 188: 58-63.

8. Yoo K-M, Moon B. Comparative carotenoid compositions during maturation and their antioxidative capacities of three citrus varieties[J]. Food Chem, 2016, 196: 544-549.

9. Yin Tingting. Comparison of fruit quality and mechanisms for differential peel color of Yellowish Ponkan and Ponkan, 2017, Zhejiang University.

10. Dhuique-Mayer C, Fanciullinc A-L, Dubois C, et al.Effect of Genotype and Environment on Citrus Juice Carotenoid Content[J]. Journal of Agricultural and Food Chemistry, 2009, 57(19): 9160-9168. 